\title{
Beat-to-beat Heart Rate detection by Smartphone's Accelerometers: validation with ECG *
}

\author{
F. Landreani, A. Martin-Yebra, C. Casellato, C. Frigo, E. Pavan, P-F Migeotte, EG. Caiani
}

Abstract - Mobile phones offer the possibility to monitor and track health parameters. Our aim was to test the feasibility and accuracy of measuring beat-to-beat heart rate using smartphone accelerometers by recording the vibrations generated by the heart during its function and transmitted to the chest wall, i.e. the so-called seismocardiographic signal (SCG). Methods: 9 healthy male volunteers were studied in supine (SUP) and in standing (ST) posture. A smartphone (iPhone6, Apple) was positioned on the thorax (POS1) to acquire SCG signal. While supine, a second smartphone was positioned on the navel (POS2). The SCG signal was recorded for 3 minutes during spontaneous respiration, synchronous with 3-leads ECG. Using a fully automated algorithm based on amplitude thresholding after rectification, the characteristic peak of the SCG signal (IVC) was detected and used to compute beat-to-beat heart duration, to be compared with the corresponding RR intervals extracted from the ECG. Results: A $\mathbf{1 0 0 \%}$ feasibility of the approach resulted for POS1 in SUP, while $89 \%$ in POS2, and $\mathbf{7 8 \%}$ for POS1 in ST. In supine, for each smartphones' position, the automated algorithm correctly identified the cardiac beats with $>98 \%$ accuracy. Linear correlation $\left(r^{2}\right)$ with $R R$ was very high $(>0.98)$ in each posture $\rightarrow$ and position, with no bias and narrow limits of agreement. Conclusions: The obtained results proved the feasibility of the proposed approach and the robustness of the applied algorithm in measuring the beat-to-beat heart rate from smartphonederived SCG, with high accuracy compared to conventional ECG-derived measure.

\section{INTRODUCTION}

Mobile devices offer the possibility to monitor and track health parameters, thanks to externally-connected devices or to embedded sensors technology. Thanks to powerful processors, extended memory, touch screen, built-in wireless connectivity, geolocalisation (i.e. location, accelerometer, and compass) and a variety of other sensors, mobile devices are continuously improving their performances and capabilities. As a result, a variety of mobile applications (apps) capable to measure parameters potentially related to healthcare through embedded sensors, interconnected devices

*Research partially supported by the Italian Space Agency (contract 2013-064-R.0, recipient E.G. Caiani), Belgian Federal Science Policy Office (BELSPO) via the ESA PRODEX program (recipient P-F Migeotte), and European Union H2020-TWINN-2015 (project "Linking excellence in biomedical knowledge and computational intelligence research for personalized management of Cardio Vascular Disease within Personalized Health Care").

F. L., A. M-Y, C.C., C.F, E.P and E.G.C. are with Politecnico di Milano, Electronic, Information and Biomedical Engineering Dpt., Milan 20133, ITALY (phone: +39.02.2399.3390; fax: +39.02.2399.3360; e-mail: enrico.caiani@polimi.it).

P.-F. is with the Université Libre de Bruxelles, Brussels, Belgium (email: pierre-francois.migeotte@ulb.ac.be). or external peripherals, is currently present on App stores for multiple platforms (iOS, Android, Windows) and continuously evolving [1].

In particular, technological improvements and miniaturization in the embedded accelerometric sensors (micro-electro mechanical system technology) allow nowadays obtaining information about cardiac activity using a mechanical signal. In particular, the vibrations generated by the heart and transmitted to the chest wall can be recorded through the mobile device accelerometers, representing the so-called seismocardiographic signal (SCG), a measure that has been recently reconsidered as a useful non-invasive technique to characterize cardiac performance [2].

Our aim was to test the feasibility and accuracy of measuring beat-to-beat interval duration using mobile device accelerometers by recording the SCG with the subject in different postures (supine and standing). In addition, while the subject was supine, the optimal position of the mobile device for optimizing signal-to-noise ratio was studied by comparing the results obtained simultaneously from two different locations on the torso.

\section{METHODS}

\section{A. Population and Experimental Set-Up}

Nine healthy right-handed male volunteers (age $25 \pm 2$, BMI $23 \pm 3 \mathrm{~kg} / \mathrm{m}^{2}$ ) were studied while in two different postures: supine and standing.

In each posture, several signals were acquired simultaneously: a) a three-lead ECG (sampling frequency, fs $=2048 \mathrm{~Hz}$ ); b) the 3-orthogonal axis SCG accelerometric signal using the smartphone mobile device (iPhone6, Apple, $\mathrm{fs}=100 \mathrm{~Hz}$, accelerometer sensitivity of $0.001 \mathrm{~g}$ ) with the app 'SensorLog'; c) a 3-component force signal (fs $=960 \mathrm{~Hz}$ ) by a dynamometric platform based on piezoelectric load cells (Type 9286B, Kistler ${ }^{\circledR}$ ).

This last signal represents the beat-to-beat time-variant reaction force of the center of mass of the subject on the platform, due to blood displacement inside the body (i.e., ballistocardiographic signal, BCG).

The ECG and BCG were synchronized by using a function generator that triggered simultaneously both systems. The BCG and SCG were triggered at start and end of acquisition by a medio-lateral impulsive force stimulus applied to the subject' shoulder, in order to introduce a spike motion artifact revealed by both the smartphone's accelerometers and the platform system (Figure 1).

In supine, where the subject was lying shirtless on a rigid support over the dynamometric platform, the acquisition protocol included three minutes at rest during spontaneous breathing with one smartphone placed on the thorax in correspondence of the midclavicular line and fourth 


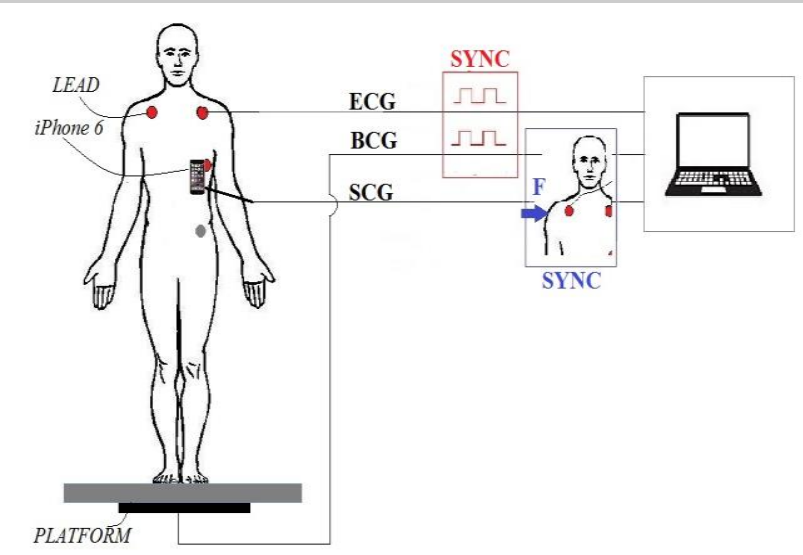

Fig. 1. Schematization of the acquisition settings in standing position: $E C G$ and BCG (from the force platform) are synchronized by a SYNC signal, while BCG and SCG (from the mobile phone) are synchronized by an impulsive force $F$ applied laterally to the subject shoulder.

intercostal space (POS1), and a second smartphone placed on the belly above the navel (POS2).

In standing, where the subject was standing barefoot on a rigid support over the dynamometric platform, the acquisition protocol included three minutes at rest during spontaneous breathing with the smartphone positioned at POS1 and kept in place by the subject using the right hand.

In both POS1 and POS2, the phone was aligned with the midclavicular line, with its top towards the head (Figure 2). In this way, the three $\mathrm{X}, \mathrm{Y}, \mathrm{Z}$ orthogonal components of the acquired SCG signal corresponded to left-to-right (L-R), head-to-foot (H-F) and antero-posterior (A-P), respectively.

The subject was asked to stay quiet, without voluntary movements, in order to ensure that recorded accelerations and forces were mainly due to mass motion inside the body. The experimental procedures involving volunteers described in this paper were in agreement with the principles outlined in the Helsinki Declaration of 1975, as revised in 2000.

\section{B. Pre-processing}

The ECG signal was processed using a wavelet-based ECG delineator [3] to derive the $\mathrm{R}$ peak positions and the beat-to-beat gold standard RR duration series.

The SCG signal was exported in a comma-separatedvalues file (.csv) and analyzed off-line using Matlab software (The Mathworks, Natick, USA).

As a first step, the ECG and BCG were synchronized taking into account the different sampling frequencies. Then, the M-L component of the SCG, where only the vibrations introduced by the impulsive force were mainly present, was used to synchronize the SCG with the BCG. As a result, SCG and ECG resulted synchronized through the BCG. As input for further processing, the A-P component was selected for POS1, while the H-F component was selected for POS2.

Due to wandering of its baseline with different influence on its components, the SCG signal was band-pass filtered (Butterworth) to remove breath motion-related artifacts: for the A-P component, a band of $5-25 \mathrm{~Hz}$ was used, while for the H-F a band of 1-25 Hz was applied. Periodic series of positive and negative deflections were clearly visible on the A-P and H-F components.

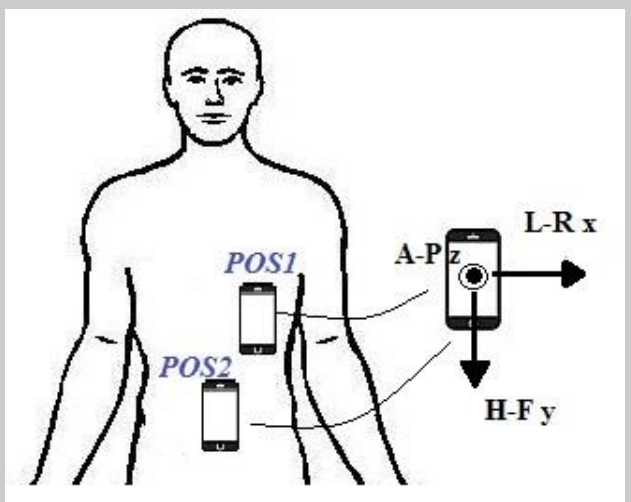

Fig. 2. Schematization of the two positions and orientation of the mobile devices used during acquisition: in supine, two smartphones were simultaneously placed in POS1 and POS2; in standing, the smartphone was kept by the subject in POS1.

In Figure 3, an example of the resulting ECG, filtered SCG A-P (POS1) and H-F (POS2) components, and H-F component of the BCG in one representative subject in supine position is shown. It is possible to observe that the morphology of SCG A-P and H-F components is different, where the SCG H-F resembles the BCG signal.

For these reasons, we decided to adopt the BCG nomenclature (I, J, K systolic waves) for the H-F component in POS2 [4], while to keep the SCG nomenclature (mitral valve closure $\mathrm{MC}$, isovolumetric contraction IVC, aortic valve opening $\mathrm{AO}$, rapid ejection $\mathrm{RE}$ ) for the A-P in POS1 [5].

\section{Fiducial point detection}

A fully automated algorithm for fiducial point detection was applied to the A-P and H-F SCG selected components, based on an evolution of the algorithm proposed in [6].

The method is summarized as follows and illustrated on Fig. 4: 1) full-wave rectification was applied, thus obtaining the entire signal with the same polarity; all peaks were identified on the rectified signal (Fig.4.a); 2) a threshold T was defined, as the half of the mean of all peaks amplitude, and only peaks whose amplitude was greater than $T$ were retained; 3) smaller peaks in the $\pm 50 \mathrm{msec}$ range of a large local peak were excluded (Fig.4.b); 4) the temporal location of the resulting peak was reported on the original signal (Fig.4.c).

As a result, the detected points on the A-P (POS1) represented the absolute minimum corresponding to the isovolumetric contraction (IVC), while on the H-F (POS2) the absolute maximum was searched around this location, thus resulting in the detection of the peak wave (J) corresponding to the end of the rapid ejection phase.

From all the detected peaks, an average template was computed considering a window spanning 50 samples before and after the peak.

Then, in order to exclude any possible misdetection point, the cross-correlation between the template and a portion of the SCG signal defined as a window centered at each detected peak, was calculated: for a correct detection, the maximum correlation value was expected to be at zero-lag. Peak detections in which the lag of the maximum correlation was more than $100 \mathrm{msec}$ were considered as false positive and automatically excluded from the further step. Finally, a $19.1 \mathrm{~mm}$ 


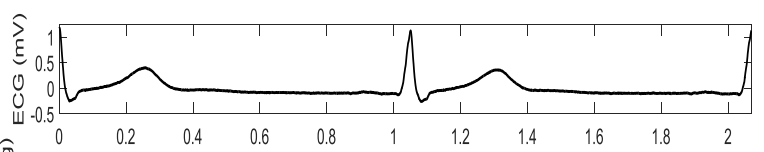

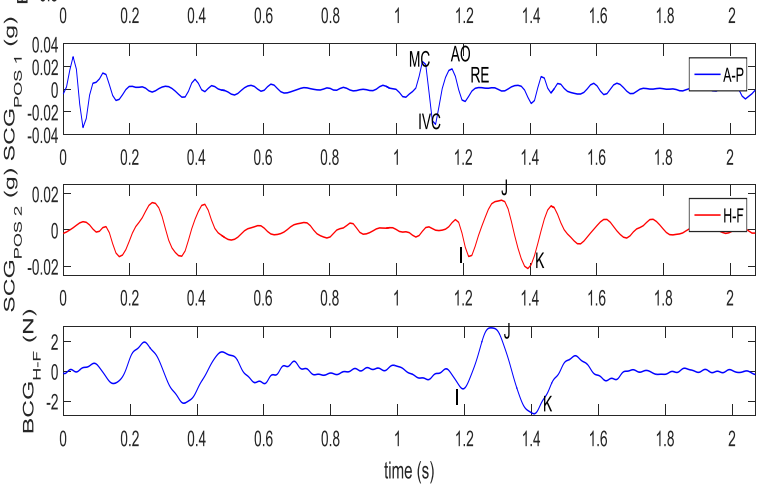

Fig. 3. Example of the ECG, simultaneous filtered SCG signals, obtained from POS1 $(A-P)$ and POS2 $(H-F)$, and BCG $(H-F)$, with the relevant adopted nomenclature.

single beat duration was identified as the peak-to-peak distance between two consecutive peaks, resulting in the IVC-IVC and J-J beat-to-beat duration time series.

\section{Validation with the gold standard and statistical analysis}

In order to test if the IVC-IVC or J-J series could represent a valid surrogate for electrodes-free heart beat duration extraction, linear correlation and Bland-Altman analysis were applied compared to the RR series extracted from the ECG, separately for supine and standing.

In order to compute the accuracy of the peak detection with superimposed the detected points were visually inspected together with the corresponding ECG, searching for missing or misdetections, separately for supine and standing.

One-way Anova for correlated samples $(\mathrm{p}<0.05)$ was applied to test the potential dependence of the accuracy of the estimate of the heart duration from the smartphone location, by comparing the RR, and the series extracted from POS1 and POS2 while in supine position.

\section{RESULTS}

Of the originally acquired 9 subjects, all the signals obtained in supine position at POS1 were retained for further processing (100\% feasibility). Conversely, at POS2 one signal was excluded for bad quality ( $89 \%$ feasibility). In standing, two signals needed to be discarded for the same reason ( $78 \%$ feasibility).

In supine, for POS1 and POS2 a total of 1874/1897 (98.6\% accuracy, 99.9\% sensitivity) and 1683/1699 (99\% accuracy, $99.9 \%$ sensitivity) peaks, respectively, were identified by the automated algorithm, one for each heartbeat in the RR, where peak detection mislocalizations were counted as false positive and missed beats as false negative. This resulted in a strong correlation with $R R\left(r^{2}>0.99\right)$ for both IVC-IVC in POS1 and J-J in POS2, not significant biases and narrow limits of agreement $( \pm 2$ SD: POS $1 \pm 20$ $\mathrm{msec}, \mathrm{POS} 2 \pm 30 \mathrm{msec})$. One-way Anova showed no significant difference between results in the two locations.
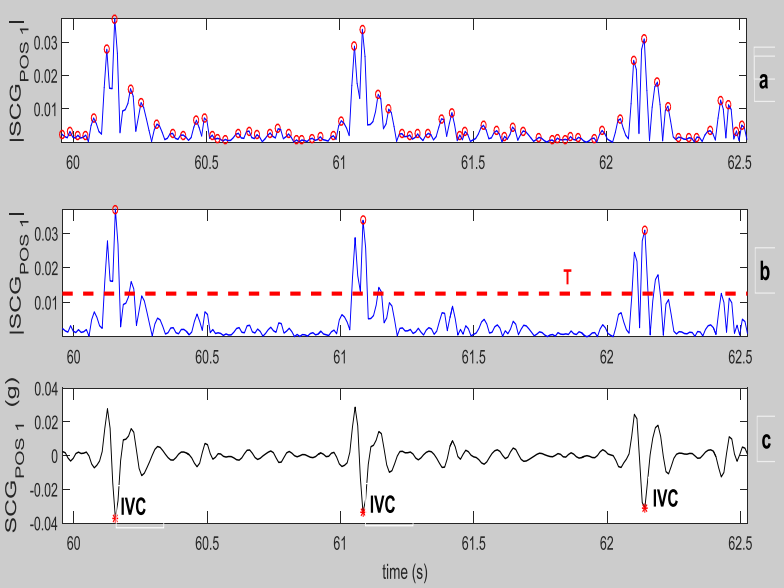

Fig. 4. Example of the steps involved in the point detection procedure applied to the A-P component of the SCG signal obtained in POS1 in supine position in a representative subject (see text for details).

In standing, for POS1 a total of 1670/1757 IVC beats (94.6\% accuracy, 99\% sensitivity) were correctly identified, one for each heartbeat. Linear correlation of IVC-IVC with RR was still high $\left(\mathrm{r}^{2}=0.989\right)$, with not significant bias and slightly larger limits of agreement $( \pm 2 \mathrm{SD}: \pm 38 \mathrm{msec})$ than in supine. Figure 5 shows the correlation and Bland Altman plots relevant to both supine and standing postures.

\section{DISCUSSION}

We tested the feasibility and the accuracy of measuring beat-to-beat heart cycle duration using mobile device accelerometers by recording the SCG with the subject in different postures (supine and standing).

Obtained results showed the feasibility of the proposed approach, in particular when the subject was supine. Additionally, two possible locations for the smartphone on the torso were tested while supine: the cardiac apex and the navel. The position in correspondence to the cardiac apex (POS1) while supine showed a $100 \%$ of feasibility, together with high correlation and slightly narrower limits of agreement in respect to POS2 and POS1 in standing. In fact, the applied algorithm to detect peak fiducial points on the SCG and compute the beat-to-beat intervals revealed high accuracy when compared to conventional ECG-derived RR series in all tested conditions, with narrow limits of agreement. In particular, optimal results were obtained while in supine with the device in POS1, with limits of agreement corresponding to $\pm 4 \mathrm{bpm}$ for the fastest heart rate analyzed (106 bpm) and to $\pm 0.6 \mathrm{bpm}$ for the lowest one (42 bpm).

Potential clinical applications of this technique could include facilitating patient engagement in the daily measure of his/her resting heart rate (RHR), to be directly collected through the use of the smartphone and automatically stored into personally controlled health record. In fact, RHR is one of the simplest cardiovascular parameters. It predicts longevity and current evidence suggests that it is also an important marker in cardiovascular diseases, including heart failure [7]. Moreover, the prognostic value of repeated RHR measurements has been strongly related to cardiovascular outcomes more than a single baseline RHR [8]. 


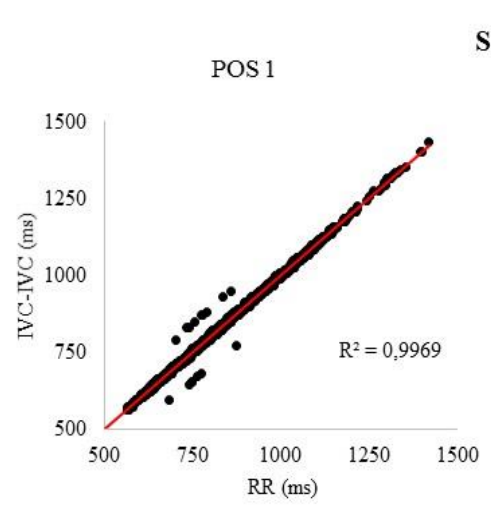

SUPINE
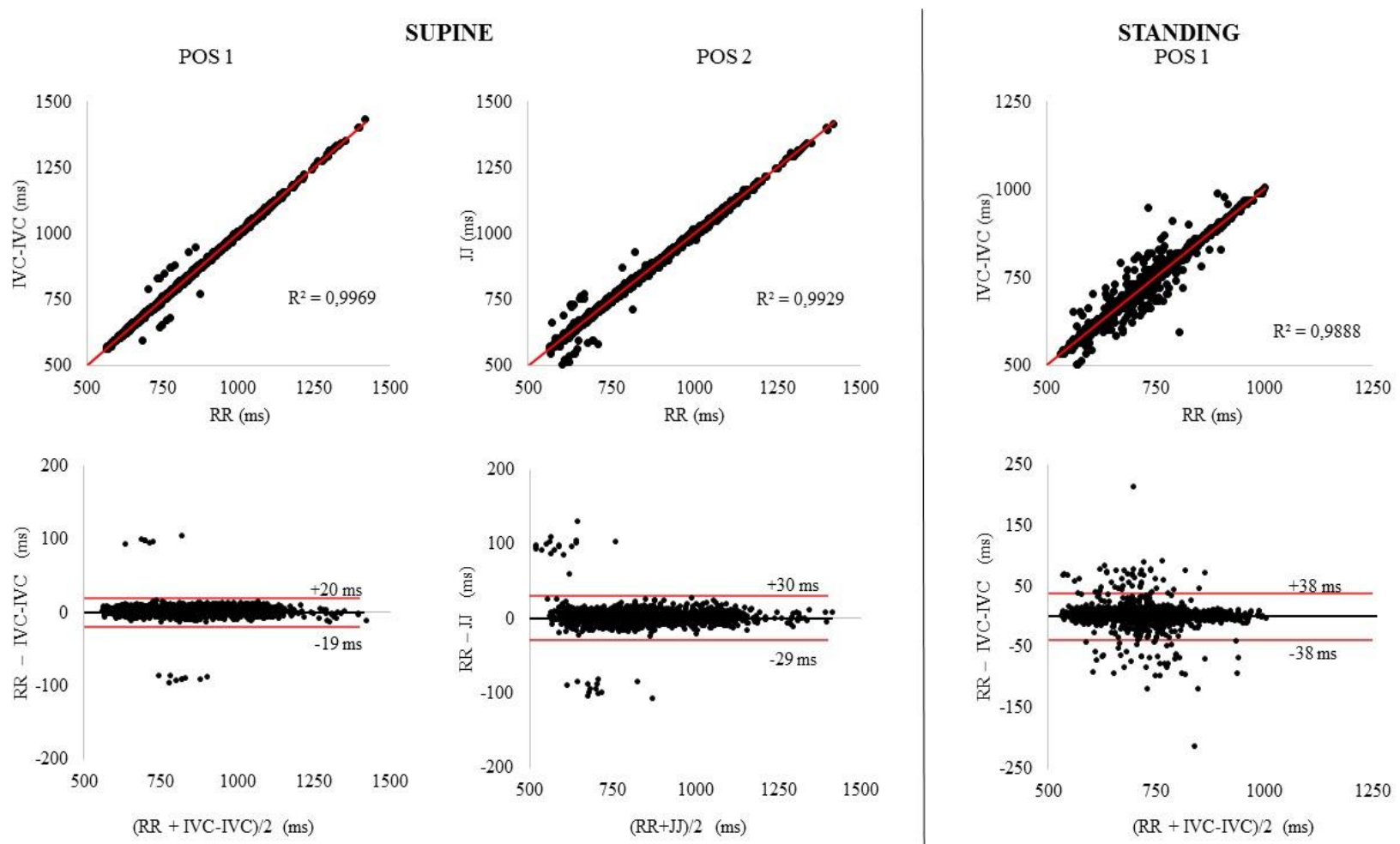

Fig. 5. Linear correlation and Bland-Altman between the gold standard RR measures from the ECG and the IVC-IVC or J-J measures from the SCG signal, in supine posture for POS1 and POS2, and in standing for POS1.

Some limitations of this study include the fact that only a limited number of male subjects of young age were tested. Additional research will be needed to confirm if the position of choice of the smartphone in correspondence of the cardiac apex is still valid for female subjects, where different anatomical features could prevent or introduce complications in the recordings, thus affecting the quality of the signal. Also, additional testing is required to evaluate feasibility of the approach in an older group of subjects, where other limitations (tremors, inability to lay on the back, etc.) could be present.

Another potential limitation could be related to the fact that all subjects were acquired in resting conditions. However, the range of explored heart rate (42-106 bpm) was quite wide, thus representing an acceptable test-bed for the evaluation of the performance of the fiducial point detection algorithm.

As a conclusion, our preliminary findings put the basis for easy, fast and accurate multiple self-evaluation of RHR using smartphone accelerometers, with potential benefits in both patient monitoring and cardiac disease prevention.

\section{REFERENCES}

[1] N. Bruning, EG Caiani, C. Chronaki, P. Guzik and E. Van der Velde, "Acquisition and analysis of cardiovascular signals on smartphones: potential, pitfalls and perspectives", Eur J Preventive Cardiol, pp. 21 (2S) 4-13, 2014

[2] O. Inan, P. Migeotte, Park, Etemadi, Tovakolian, Casanella, Zanetti, Funtova, Prisk and Rienzo, "Ballistocardiography and Seismocardiography: a review of Recent Advances" IEEE, Journal of Biomedical an Health Informatics, 2014
[3] J. Martinez, R. Almeida, S. Olmos, A. Rocha e P. Laguna, "Waveletbased ECG delineator: evaluation on standard database", IEEE Trans Biomed Eng, vol. 51, pp. 570-581, 2004

[4] W. R Scarborough \& S. A. Talbot, "Proposals for ballistocardiographic nomenclature and conventions - Revised and extended report of Committee on Ballistocardiographic Terminology", Circulation, vol. 14, pp. 435-450, 1956

[5] R. S Crow, P. Hannan, D. Jacobs, L. Hedquist \& Salerno, "Relationship between seismocardiogram and echocardiogram for events in the cardiac cycle." American Journal of Noninvasive Cardiology, vol. 8(1), 39-46, 1994

[6] J. Ramos-Castro, J. Moreno, H. Miranda-Vidal, M.A. García González, Mireya Fernández-Chimeno, G. Rodas and Ll Capdevila., "Hear rate Variability analysis using a Seismocardiogram signal" EMBC, Annual International Conference of the IEEE, pp. 5642-5645, 2012

[7] M. Bohm, J. C. Reil, P. Deedwania, J. Kim e J. Borer, "Resting heart rate: risk indicator and emerging risk factor in cardiovascular disease" American Journal of Medicine, vol. 128, n. 3, pp. 219-28, 2015.

[8] V. Hamill, I. Ford, Fox, M. Böhm, J. Borer, R. Ferrari, Komajda, Steg, L. Tavazzi, Tendera e K. Swedberg, "Repeated Heart Rate Measurement and Cardiovascular Outcomes in Left Ventricular Systolic Dysfunction.” American Journal of Medicine, vol. 128, n. 10 pp. 1102- 1108, 2015. 